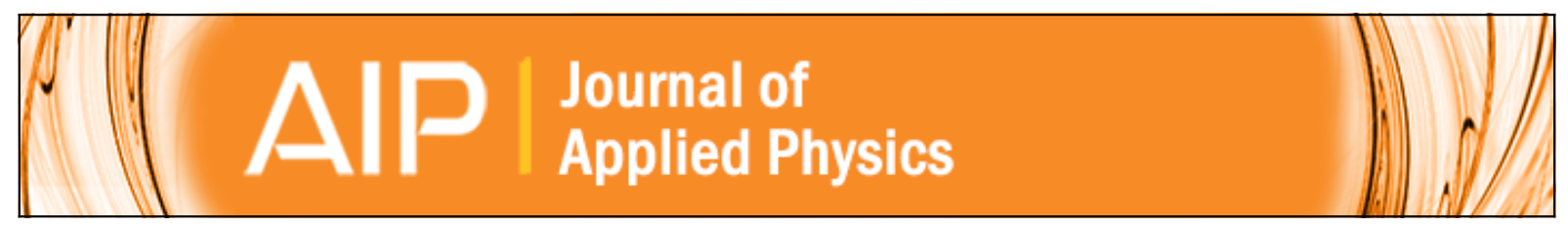

\title{
Detection of the onset of nanocrystallization by calorimetric and magnetic measurements
}

\author{
J. S. Blázquez, V. Franco, C. F. Conde, A. Conde, and S. Roth
}

Citation: Journal of Applied Physics 97, 044308 (2005); doi: 10.1063/1.1849826

View online: http://dx.doi.org/10.1063/1.1849826

View Table of Contents: http://scitation.aip.org/content/aip/journal/jap/97/4?ver=pdfcov

Published by the AIP Publishing

\section{Articles you may be interested in}

Magnetic permeability of Si-rich (FeCoNi)-based nanocrystalline alloy: Thermal stability in a wide temperature range

J. Appl. Phys. 113, 17A310 (2013); 10.1063/1.4794718

Magnetic field driving custom assembly in (FeCo) nanocrystals

Appl. Phys. Lett. 89, 033508 (2006); 10.1063/1.2222254

Structural, magnetic, and magnetostriction behaviors during the nanocrystallization of the amorphous Ni $5 \mathrm{Fe}$ 68.5 Si 13.5 B 9 Nb 3 Cu 1 alloy

J. Appl. Phys. 99, 08F104 (2006); 10.1063/1.2162810

Thermomagnetic detection of recrystallization in $\mathrm{FeCoNbBCu}$ nanocrystalline alloys

Appl. Phys. Lett. 79, 2898 (2001); 10.1063/1.1413957

Effects of Co addition on magnetic properties and nanocrystallization in amorphous Fe $84 \mathrm{Zr} 3.5 \mathrm{Nb} 3.5 \mathrm{~B} 8 \mathrm{Cu} 1$ alloy

J. Appl. Phys. 86, 6301 (1999); 10.1063/1.371690

\section{ФSHIMADZU Powerful, Multi-functional UV-Vis-NIR and Excellence in science FTIR Spectrophotometers}

Providing the utmost in sensitivity, accuracy and resolution for applications in materials characterization and nano research

- Photovoltaics

- Polymers

- Thin films

- Paints

- Ceramics

- DNA film structures

- Coatings

- Packaging materials
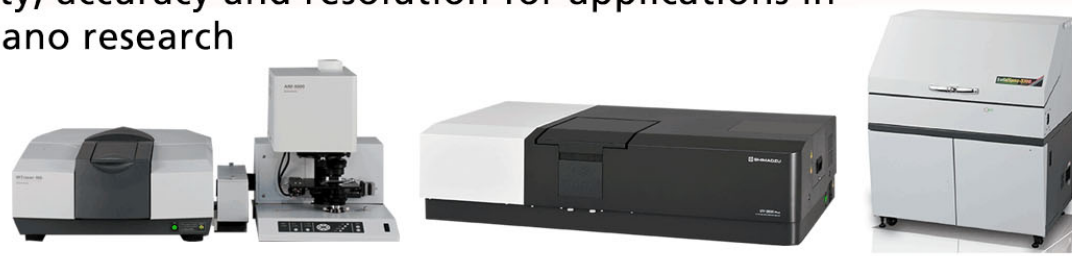


\title{
Detection of the onset of nanocrystallization by calorimetric and magnetic measurements
}

\author{
J. S. Blázquez \\ Departamento de Física de la Materia Condensada, Instituto de Ciencia de Materiales de Sevilla-Consejo \\ Superior de Investigaciones Cientifícas (ICMSE-CSIC), Universidad de Sevilla, P.O. Box 1065, 41080 \\ Sevilla, Spain and Leibniz Institute for Solid State and Materials Research Dresden (IFW-Dresden), Institute \\ for Metallic Materials, Helmholtzstrasse 20, 01069 Dresden, Germany \\ V. Franco, C. F. Conde, and A. Conde ${ }^{\text {a) }}$ \\ Departamento de Física de la Materia Condensada, Instituto de Ciencia de Materiales de Sevilla-Consejo \\ Superior de Investigaciones Cientifícas (ICMSE-CSIC), Universidad de Sevilla, P.O. Box 1065, 41080 \\ Sevilla, Spain

\section{S. Roth} \\ Leibniz Institute for Solid State and Materials Research Dresden (IFW-Dresden), Institute for Metallic \\ Materials, Helmholtzstrasse 20, 01069 Dresden, Germany
}

(Received 13 July 2004; accepted 22 November 2004; published online 24 January 2005)

\begin{abstract}
It is generally accepted that measurements of the magnetic properties are more sensitive than measurements of the enthalpy changes in the detection of the onset of crystallization of ferromagnetic phases emerging from a paramagnetic amorphous alloy. In this work, it is shown that the formation of a very fine nanocrystalline microstructure can make this assumption incorrect. Under some circumstances, the nanocrystallization onset temperature obtained from magnetic techniques is higher than the one obtained from enthalpy changes. The phenomenon is explained in terms of the superparamagnetic behavior of the uncoupled nanocrystals at the very early stages of nanocrystallization. @ 2005 American Institute of Physics. [DOI: 10.1063/1.1849826]
\end{abstract}

\section{INTRODUCTION}

A widespread technique to obtain nanocrystalline alloys is the controlled partial crystallization of a precursor amorphous alloy with a suitable composition. The optimization of the material properties requires an accurate determination of the onset temperature of nanocrystallization, $T_{X}$. This parameter, which depends on the heating rate due to the thermally activated character of the devitrification process, can be directly measured from enthalpy changes. However, indirect measurements, like changes in the electrical resistivity or in the magnetic properties due to the formation of a new phase, are claimed to be more sensitive than calorimetric measurements for the detection of the emerging crystals. ${ }^{1}$ This attributed higher sensitivity is in agreement with the fact that the onset temperature extracted from magnetic techniques $\left(T_{X}^{\mathrm{mag}}\right)$ is usually lower than the one obtained from differential scanning calorimetry $\left(T_{X}^{\mathrm{DSC}}\right) .{ }^{1}$ Magnetization measurements are especially useful when $T_{X}$ is above the Curie temperature $T_{C}$ of the amorphous phase. In this case, a zero signal is obtained previous to the onset of crystallization and the signal increases as the crystalline volume fraction $X$ increases, allowing detailed kinetic studies of the transformation. ${ }^{2}$

In this paper we report experimental results indicating that, under some circumstances, the assumption $T_{X}^{\mathrm{mag}} \leqslant T_{X}^{\mathrm{DSC}}$ is no longer true. For this purpose, Fe-based nanocrystalline alloys have been studied. Their characteristic microstructure consists of randomly dispersed crystallites $(\sim 10 \mathrm{~nm})$ of a

\footnotetext{
a) Author to whom correspondence should be addressed; FAX: +34-95/4612097; electronic mail: conde@us.es
}

ferromagnetic phase ( $\alpha$-Fe-type) embedded in a residual amorphous matrix, also ferromagnetic but with a lower $T_{C}{ }^{3}$ This microstructure facilitates the averaging of the magnetocrystalline anisotropy due to the exchange coupling of the nanocrystals via the ferromagnetic amorphous matrix. The reduced value of the overall anisotropy is responsible for the outstanding soft magnetic properties exhibited by these alloys. ${ }^{4}$ The difference in the nanocrystallization onset temperatures obtained from the calorimetric and magnetic techniques will be analyzed taking into account the possibility of a superparamagnetic behavior of the emerging monodomain nanoparticles embedded in the paramagnetic amorphous matrix.

\section{EXPERIMENT}

Samples of $\quad \mathrm{Fe}_{60} \mathrm{Co}_{18} \mathrm{Nb}_{6} \mathrm{~B}_{15} \mathrm{Cu}_{1}, \quad \mathrm{Fe}_{60} \mathrm{Co}_{18} \mathrm{Nb}_{6} \mathrm{~B}_{16}$ (Hitperm-type alloys), and $\mathrm{Fe}_{74} \mathrm{Si}_{16} \mathrm{Nb}_{3} \mathrm{~B}_{6} \mathrm{Cu}_{1}$ (Finemet-type) were studied. They will be denoted in the following as $\mathrm{Cu}-\mathrm{HT}, \mathrm{HT}$, and FM, respectively. Ribbons 5-10 mm wide and $10-20 \mu \mathrm{m}$ thick were produced by melt spinning. $T_{X}^{\mathrm{DSC}}$ was measured by differential scanning calorimetry (DSC) in a Perkin-Elmer DSC7 calorimeter; $T_{X}^{\mathrm{mag}}$ was measured by thermomagnetic gravimetry (TMG) in a Perkin-Elmer TGA7 thermobalance using a small magnet (maximum field $\sim 20 \mathrm{mT}$ ). Both measurements were performed at heating rates of $10 \mathrm{~K} / \mathrm{min}$. A vibrating-sample magnotemeter (VSM) was used to study quantitatively the dependence of the magnetic signal with the applied magnetic field $B=0.002,0.02$, and $0.2 \mathrm{~T}$, using a heating rate of $5 \mathrm{~K} / \mathrm{min}$. 


\section{RESULTS}

The microstructure of the $\mathrm{Cu}-\mathrm{HT}$ and $\mathrm{HT}$ alloys is similar. Both develop a nanocrystalline $\alpha$-Fe, Co phase and present the same crystalline volume fraction at the end of the nanocrystallization process $(X \sim 0.65){ }^{5}$ However, the addition of 1 at. \% of $\mathrm{Cu}$ in the $\mathrm{Cu}-\mathrm{HT}$ alloy with respect to the HT alloy provokes a strong refinement of the microstructure through an enhancement of the heterogeneous nucleation due to the formation of $\mathrm{Cu}$ clusters before nanocrystallization. ${ }^{6}$ Consequently, the mean grain sizes are very different: $D$ $\sim 5 \mathrm{~nm}$ for $\mathrm{Cu}-\mathrm{HT}$ and $\sim 20 \mathrm{~nm}$ for $\mathrm{HT}^{7}$ In the case of the FM alloy, the $\mathrm{Cu}$-clustering phenomenon is also present, and the mean grain size of the $\alpha-\mathrm{Fe}, \mathrm{Si}$ phase at the end of nanocrystallization is $\sim 15 \mathrm{~nm}^{2}$ Although the $\mathrm{Cu}$-clustering phenomenon occurs in both the FM and $\mathrm{Cu}-\mathrm{HT}$ alloys, a larger grain size is obtained in the case of $\mathrm{FM}^{8}{ }^{8} \mathrm{~A}$ lower $\mathrm{Nb}$ content (3 at. \%) with respect to that of $\mathrm{Cu}-\mathrm{HT}(6$ at. \%) and the presence of $\mathrm{Si}$ in the $\alpha$-Fe crystals could be the origin of this larger grain growth in the FM alloys.

In order to calculate the crystalline volume fraction, both calorimetric and magnetic measurements can be used. In the first case, the crystalline volume fraction $X$ can be associated with the enthalpy change $(X \propto \Delta H)$, while for magnetic measurements it is connected to the magnetization $(X \propto M)$. In fact, the magnetization of the nanocrystals depends on the temperature but, in the case of isothermal experiments or for small temperature ranges (provided that the Curie temperature of the phase is distant enough), reasonable results can be obtained by considering a temperature-independent magnetization of the phase. Under these conditions it can be assumed that the crystalline fraction is directly proportional to the magnetization signal. Nevertheless, the relationship involving enthalpy and crystalline fraction is also not exempt from criticisms, ${ }^{9}$ as it disregards the continuous compositional change of the amorphous matrix along the nanocrystallization process. The signal measured in a DSC run corresponds to the time variation of the enthalpy of the system during the transformation, $d H / d t$.

Both techniques, TMG and DSC, allow a very good control of the heating rate. Therefore, a comparison between $T_{X}$ obtained at the same heating rate will give an idea of the sensitivity of the technique without being affected by the thermally activated character of the process. Figure 1 shows the normalized DSC plots (peak area $=1$ ) of the three as-cast alloys, together with the normalized first derivative of the TMG curves (at a field of $\sim 20 \mathrm{mT}$ ) with respect to the temperature (the noise in these curves is due to the numerical derivatives). The onset of crystallization is detected at a lower temperature by TMG than by DSC. However, whereas the difference in $T_{X}$ between both techniques is only of $3 \mathrm{~K}$ for the $\mathrm{Cu}-\mathrm{HT}$ alloy, that difference clearly increases for the other two alloys, being $>10 \mathrm{~K}$.

To explain this effect, the $\mathrm{Cu}$-clustering process before nanocrystallization in the alloys with $\mathrm{Cu}$ could be taken into account. The formation of this $\mathrm{Cu}$-rich nonmagnetic phase implies that an extra amount of enthalpy change would be observed at a lower temperature. This could make the onset temperatures detected from DSC and TMG closer, as found

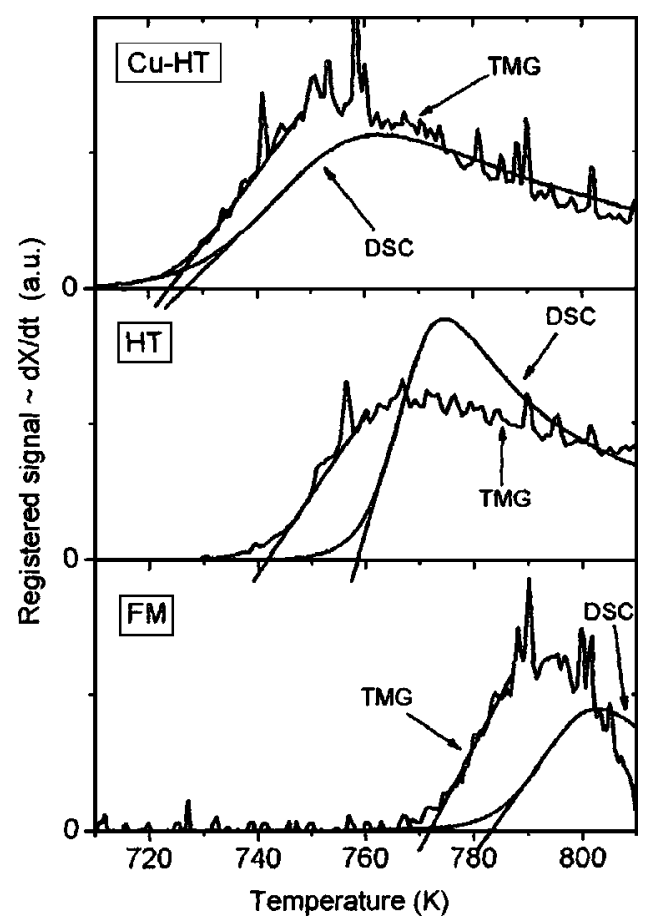

FIG. 1. Thermomagnetic gravimetry (TMG) $(d M / d t)$ and differential scanning calorimetry (DSC) $(d H / d t)$ plots at a heating rate of $10 \mathrm{~K} / \mathrm{min}$. The TMG signal is obtained as the derivative of the experimental $M(T)$ data for an applied field of $\sim 0.02 \mathrm{~T}$.

for the $\mathrm{Cu}-\mathrm{HT}$ alloy. However, it cannot explain the difference between the $T_{X}$ values observed by DSC and TMG for the FM alloy, for which an even higher density of $\mathrm{Cu}$ clusters than for the $\mathrm{Cu}-\mathrm{HT}$ alloy is expected. ${ }^{10}$

To investigate these differences in the crystallization onset detected by DSC and TMG techniques for the studied alloys, the applied magnetic field in the TMG measurements was changed to test its influence on the observed value of $T_{X}^{\mathrm{mag}}$. Table I shows $T_{X}$ values obtained by DSC and by TMG using two applied fields controlled by the position of the magnet at the thermobalance: the maximum achievable field (high) and a field which supplies a signal equal to $10 \%$ of the maximum signal (low). It can be observed that whereas for the HT and FM alloys $T_{X}^{\mathrm{mag}}$ is almost independent of the field, for the Cu-HT alloy, $T_{X}^{\text {mag }}$ is higher for low-field measurements than for high-field ones. In the case of low-field measurement, $T_{X}^{\mathrm{mag}}$ is even higher than that observed from the DSC measurements.

A quantitative study of the field dependence of $T_{X}^{\mathrm{mag}}$ was done using a VSM. Figure 2 shows $M$ versus temperature plots for the $\mathrm{Cu}-\mathrm{HT}$ and $\mathrm{HT}$ alloys obtained for different

TABLE I. Values of $T_{X}$ measured from DSC and TMG techniques at the same heating rate, $10 \mathrm{~K} / \mathrm{min}$. High field corresponds to the positioning of the magnet to obtain the maximum signal at room temperature $(\sim 20 \mathrm{mT})$. Low field is obtained for a signal equal to $10 \%$ of that maximum.

\begin{tabular}{ccccc}
\hline \hline Alloy & Composition (at. \%) & $T_{X}^{\text {DSC }}( \pm 1 \mathrm{~K})$ & $\begin{array}{c}T_{X}^{\text {mag }}( \pm 2 \mathrm{~K}) \\
\text { (high field) }\end{array}$ & $\begin{array}{c}T_{X}^{\text {mag }}( \pm 2 \mathrm{~K}) \\
\text { (low field) }\end{array}$ \\
\hline $\mathrm{Cu}-\mathrm{HT}$ & $\mathrm{Fe}_{60} \mathrm{Co}_{18} \mathrm{Nb}_{6} \mathrm{~B}_{15} \mathrm{Cu}_{1}$ & 727 & 724 & 731 \\
$\mathrm{HT}$ & $\mathrm{Fe}_{60} \mathrm{Co}_{18} \mathrm{Nb}_{6} \mathrm{~B}_{16}$ & 759 & 741 & 740 \\
FM & $\mathrm{Fe}_{74} \mathrm{Si}_{16} \mathrm{Nb}_{3} \mathrm{~B}_{6} \mathrm{Cu}_{1}$ & 784 & 771 & 769 \\
\hline \hline
\end{tabular}




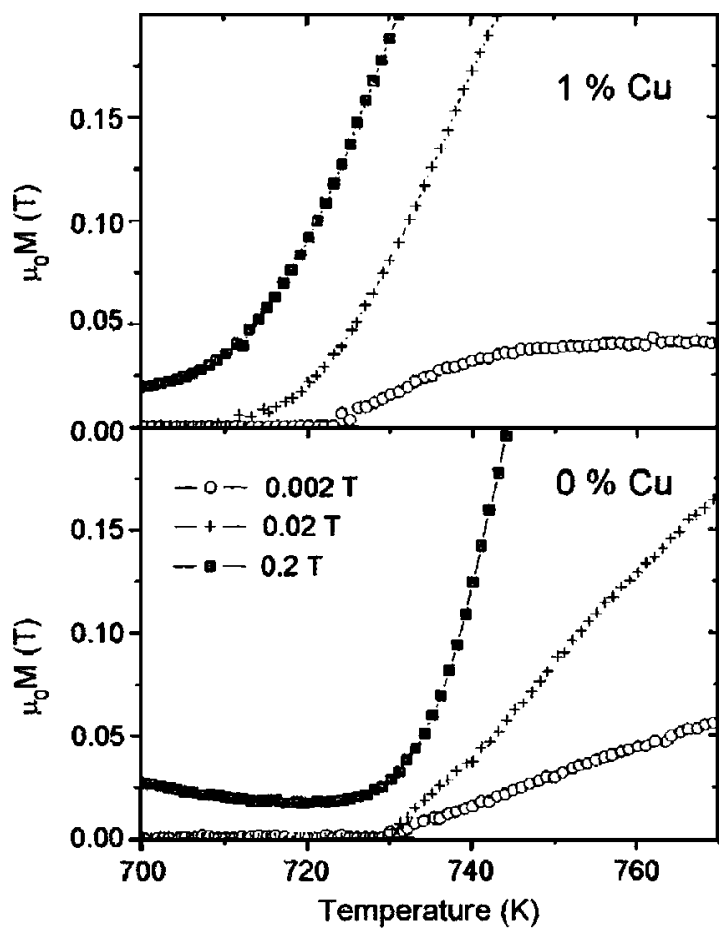

FIG. 2. Magnetization $M(T)$ curves for $\mathrm{Fe}_{60} \mathrm{Co}_{18} \mathrm{Nb}_{6} \mathrm{~B}_{16-y} \mathrm{Cu}_{y}(y=0,1)$ alloys at a heating rate of $\sim 5 \mathrm{~K} / \mathrm{min}$ registered in the vibrating-sample magnetometer (VSM) at the early stages of nanocrystallization using different applied fields.

applied magnetic fields. Samples were heated at $\sim 5 \mathrm{~K} / \mathrm{min}$. For the highest field $(B=0.2 \mathrm{~T})$, even the paramagnetic matrix gives a nonzero signal. However, it is possible to distinguish a temperature at which the signal rises due to the onset of nanocrystallization. In the case of the alloy without $\mathrm{Cu}$, $T_{X}^{\mathrm{mag}}=730 \pm 1 \mathrm{~K}$ and is independent of the applied field. On the other hand, for the alloy with $\mathrm{Cu}, T_{X}^{\mathrm{mag}}$ increases as the applied field is decreased: $T_{X}^{\mathrm{mag}}=707,712$, and $722 \pm 1 \mathrm{~K}$ for $B=0.2,0.02$, and $0.002 \mathrm{~T}$, respectively. At first sight, this increase could be related to the sensitivity of the measuring equipment. However, this can be ruled out as the signal level for a given field value is the same for both the $\mathrm{Cu}-\mathrm{HT}$ and HT samples and the $T_{X}$ increase as the applied field decreases is only observed for the $\mathrm{Cu}-\mathrm{HT}$ samples.

\section{DISCUSSION}

An explanation of this effect may be found in the differences observed in the microstructure of the studied alloys. At the very beginning of nanocrystallization, very small crystals are formed, which can be considered isolated and, therefore, in a superparamagnetic state. The magnetization produced by a monodispersed superparamagnetic system is described by

$$
M=N_{\nu} m L\left(m B / k_{B} T\right),
$$

where $N_{\nu}$ is the numerical density of particles, $m$ the magnetic moment of the individual particles, $B$ the magnetic field, $T$ the temperature, $k_{B}$ the Boltzmann constant, and $L$ the Langevin function.

Several assumptions may be made to have a view of the existing microstructure at the very early stages of nanocrystallization. A first approximation would be to consider instan- taneous nucleation regime, i.e., the number of nanocrystals remains constant during the nanocrystallization. After this, it is easy to obtain the value of $N_{\nu}$ for each alloy: 0.01 and $0.00016 \mathrm{~nm}^{-3}$ for the $\mathrm{Cu}-\mathrm{HT}$ and HT alloys, respectively, which yields $X \sim 65 \%$ for a value of $D$ of 5 and $20 \mathrm{~nm}$, respectively. The value of $m$ can be obtained as the number of atoms in the nanocrystal times its atomic magnetic moment, which can be estimated as $2.2 \mu_{B}$. The bcc unit cell contains two atoms in a volume equal to $a^{3}$, where $a$ $=0.28625 \mathrm{~nm}$ is the lattice parameter ${ }^{10}{ }^{1}$ therefore,

$$
m(D)=2.2 \mu_{B} \frac{2}{a^{3}} \frac{\pi D^{3}}{6} .
$$

At this point, magnetization can be obtained as a function of the grain size using Eq. (1), where $N_{\nu}$ would characterize the microstructure of each particular case. The next step will be to relate the grain size $D$ to the temperature. This can be done using the known kinetic parameters of these alloys $^{11,12}$ and the Avrami equation. Although this equation is valid for isothermal regimes, we are only interested in a very small temperature range, just after the onset of crystallization. Therefore, kinetic parameters will be used in the following without considering their thermal dependence.

The Avrami equation establishes a relationship between the fraction of the process, $X^{*}$, and the time, $t$,

$$
X^{*}=1-\exp \left[-(K t)^{n}\right],
$$

where $K$ is the frequency factor and $n$ is the Avrami exponent. The Avrami exponent is approximately 1 and is independent of the temperature at the early stages of nanocrystallization. ${ }^{12} K$ follows an Arrhenius-type dependence with the temperature,

$$
K=K_{0} \exp \left(-Q / k_{B} T\right),
$$

where $K_{0}$ is a constant and $Q$ is an activation energy. From the kinetic results, $K_{0} \sim 10^{13} \mathrm{~s}^{-1}$ and $Q \sim 2.4 \mathrm{eV} .{ }^{12}$ In the aim of simplifying the calculations and taking into account that we are interested in a very small temperature range, the thermal dependence of $K$ has been neglected and a value of $\sim 0.0004 \mathrm{~s}^{-1}$ (at $T=725 \mathrm{~K}$ ) has been used. $X^{*}=X / 0.65$ for both the studied alloys, thus $X^{*}=1$ at the end of the nanocrystallization. From $t=\left(T-T_{X}\right) / \beta$, and taking into account (2) and (3),

$$
T=T_{X}-\frac{\beta}{K} \ln \left(1-\frac{1}{0.65} N_{\nu} \frac{\pi}{6} D^{3}\right),
$$

where $T_{X}$ is $710 \mathrm{~K}$ for the $\mathrm{Cu}-\mathrm{HT}$ alloy and $730 \mathrm{~K}$ for the HT alloy, and $\beta=5 \mathrm{~K} / \mathrm{min}$, the same value as used in the VSM experiments.

Considering a sensitivity of the VSM device of $0.1 \mathrm{memu}$, the detection limit of the magnetization will be about $0.1 \mathrm{mT}$ in the case of the studied samples (sample mass $\sim 8 \mathrm{mg}$, density $\sim 7.8 \mathrm{~g} / \mathrm{cm}^{3}$ ). Figure 3 shows the effect of the applied field on the detected crystallization onset as is expected under the assumptions indicated above; experimental values from Fig. 2 are also included. It can be seen that the observed behavior is qualitatively reproduced. An important effect of the applied magnetic field is found for 


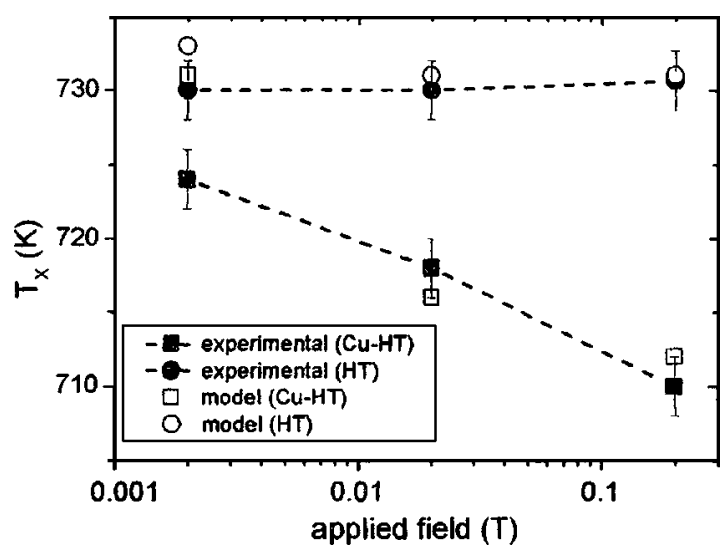

FIG. 3. Experimental $T_{X}^{\mathrm{mag}}$ obtained by vibrating-sample magnotemeter (VSM) measurements (solid symbols) and results of the modellization (hollow symbols) of $\mathrm{Fe}_{60} \mathrm{Co}_{18} \mathrm{Nb}_{6} \mathrm{~B}_{16-y} \mathrm{Cu}_{y}(y=0,1)$ alloys as noninteracting monodispersed superparamagnetic particles (numerical densities $N_{\nu}=0.01$ and $0.00016 \mathrm{~nm}^{-3}$ and crystallization onset temperature $T_{X}=710$ and $730 \mathrm{~K}$ for the alloys with and without $\mathrm{Cu}$, respectively). The experimental symbols are joined by lines as a guide to the eye.

the $\mathrm{Cu}-\mathrm{HT}$ sample (with a much lower grain size than $\mathrm{HT}$ ): the detection limit for $0.2 \mathrm{~T}$ is overcome at a temperature $18 \mathrm{~K}$ lower than for $0.002 \mathrm{~T}$. However, for the HT sample this difference is smaller than $2 \mathrm{~K}$. In terms of crystalline volume fraction, for the HT sample the detection limit would be overcome for $X<2 \%$ even for the lowest applied field $(0.002 \mathrm{~T})$. However, in the case of the Cu-HT alloy at $B$ $=0.002 \mathrm{~T}$, the detection limit would be overcome only for $X>15 \%$, which could explain why the apparent onset of crystallization is detected in this case even later than using DSC (Table I).

Although strong assumptions were made to model the $M$ signal, the trends observed experimentally are described with a fairly good qualitative agreement. It must be taken into account that the approximations used above lose their validity as the difference in temperature between the detected onsets becomes larger: the isothermal kinetic parameters will be modified and, at a high crystalline volume fraction, superparamagnetic particles will interact. ${ }^{13}$ These effects could explain the overestimated modeled value of the crystallization onset for the $\mathrm{Cu}-\mathrm{HT}$ alloy at $0.002 \mathrm{~T}$ with respect to the experimental value. As the range of temperatures is extended, the temperature dependence of $K$ should be taken into account. On the other hand, as the crystalline volume fraction increases, the interaction among the particles will modify the magnetic response of the system from the simple Langevin function used in this work. Nevertheless, the observed trend is successfully described.

\section{CONCLUSIONS}

In conclusion, for a comparison of calorimetric and magnetic techniques as tools for detecting the crystallization onset, it has to be taken into account that the sensitivity of the latter can be dependent, in some cases, on the applied magnetic field. The generally accepted idea that magnetic techniques have a higher sensitivity than the calorimetric ones is not necessarily true in presence of a very fine microstructure, which can produce a superparamagnetic behavior.

\section{ACKNOWLEDGMENTS}

This work was supported by the CICYT of the Spanish Government and EU FEDER (Project MAT 2001-3175), and the PAI of the Regional Government of Andalucía. One of the authors (J.S.B.) acknowledges a research contract of that regional government.

${ }^{1}$ W. Hofstetter, H. Sassik, R. Grössinger, R. Trausmuth, G. Vertesy, and L. F. Kiss, Mater. Sci. Eng., A 226-228, 213 (1997).

${ }^{2}$ C. F. Conde and A. Conde, Mater. Lett. 21, 409 (1994).

${ }^{3}$ M. E. McHenry, M. A. Willard, and D. E. Laughlin, Prog. Mater. Sci. 44, 291 (1999).

${ }^{4}$ A. Hernando, M. Vázquez, T. Kulik, and C. Prados, Phys. Rev. B 51, 3581 (1995).

${ }^{5}$ J. S. Blázquez, V. Franco, C. F. Conde, and A. Conde, J. Magn. Magn. Mater. 254-255, 460 (2003).

${ }^{6}$ Y. Zhang, J. S. Blázquez, A. Conde, P. J. Warren, and A. Cerezo, Mater. Sci. Eng., A 353, 158 (2003).

${ }^{7}$ J. S. Blázquez, V. Franco, and A. Conde, J. Phys.: Condens. Matter 14, 11717 (2002).

${ }^{8}$ J. S. Blázquez, J. M. Borrego, C. F. Conde, A. Conde, and J. M. Greneche, J. Phys.: Condens. Matter 15, 3957 (2003).

${ }^{9}$ J. M. Barandiarán, I. Tellería, J. S. Garitaonandia, and H. A. Davies, J. Non-Cryst. Solids 329, 57 (2003).

${ }^{10}$ M. Ohnuma, D. H. Ping, T. Abe, H. Onodera, K. Hono, and Y. Yoshizawa, J. Appl. Phys. 93, 9186 (2003).

${ }^{11}$ J. S. Blázquez, C. F. Conde, and A. Conde, J. Non-Cryst. Solids 287, 187 (2001).

${ }^{12}$ J. S. Blázquez, C. F. Conde, and A. Conde, Appl. Phys. A: Mater. Sci. Process. 76, 571 (2003).

${ }^{13}$ V. Franco, L. F. Kiss, T. Kemény, I. Vincze, C. F. Conde, and A. Conde, Phys. Rev. B 66, 224418 (2002). 